\title{
USING A SMART RECOGNITION FRAMEWORK FOR THE AUTOMATED TRANSFER OF STRUCTURAL WHOLE ENGINE MODELS
}

\author{
BENJAMIN SPIESS ${ }^{1}$, KLAUS HÖSCHLER ${ }^{2}$ AND MAREN FANTER $^{3}$ \\ ${ }^{1}$ Doctoral Candidate, Chair of Aero Engine Design \\ Siemens-Halske-Ring 14, 03046 Cottbus \\ spiess.benjamin@b-tu.de \\ ${ }^{2}$ Chair holder, Chair of Aero Engine Design \\ Siemens-Halske-Ring 14, 03046 Cottbus \\ klaus.hoeschler@b-tu.de \\ ${ }^{3}$ Rolls-Royce Deutschland GmbH \\ Eschenweg 11, 15827 Blankenfelde-Mahlow \\ maren.fanter@rolls-royce.com
}

Key words: Recognition, CAD, FEM

\begin{abstract}
The development of adequate simulation models from geometric CAD assemblies is one of the most important tasks in early design phases. With this step requiring a lot of manual effort, the desire for a process efficiency improvement via an automated solution rises. In order to derive information about the assembly to build Finite-Element (FE) models, various different steps have to be taken which require visual assessment and engineering evaluation, knowledge and judgement. The approach described in this research mimics the engineer's logic and way of thinking to automate these steps. Thereof, the recognition of entities plays a fundamental role for further processing. To achieve the desired recognition, methods have been developed to retrieve criteria like form, function, context and positioning from the available geometry data. The developed recognition framework supports and provides a component categorization so that specifically optimized process chains for each category can be implemented, depicting a more robust and reasonable overall process.
\end{abstract}

\section{Introduction}

Simulations of structures play an essential role during design and development phases of products of various kind. These simulations help to estimate the behaviour of a component, predict its dynamic characteristics and evaluate its durability. The outcome of those studies is the basis for design improvements, safety or limit conditions evaluations. The most common simulation technique in general and especially in the field of aero engine development is the Finite Element Method (FEM). Simple and single components do not require a lot of effort to be converted to a FE model because of little or no boundary conditions. However, to derive a simulation model from a complex Computer-Aided-Design (CAD) assembly numerous time-consuming and manual tedious tasks have to be executed. Due to the effort required to transfer the geometric model to a structural FE analysis model, extensive design studies 
are not practical and thus, the demand for a better integrated and automated solution rises.

To meet this challenge, Hamri et al. [1] propose an intermediate model between the CAD and CAE model to keep the link from the FE model and results to the initial geometry. Nevertheless, in case of a change of properties and interfaces maintaining the correct boundary conditions in the updated simulation model is rather impossible in complex assemblies. In a later work [2], methods, models and tools are investigated in order to automate the CAD pre-processing for simulation purposes but applications on complex assemblies like aero engine models do still imply issues. In [3], a similar path is pursued by evaluating a CAD-CAE integration by using a top-level integrated model being parent to both CAD and CAE model. Interface and feature information is stored in this model and basis for idealizations like detail removal or dimensional reduction. The main focus is put on single components, thus the change of interfaces and boundary conditions in assemblies is not directly accessed.

The application of boundary conditions, simplification of components while maintaining a satisfactory compromise between model accuracy and detail [4] and a well-founded set-up of FE properties are exemplary tasks which the engineering team has to accomplish. Furthermore, a general and direct CAD to FE conversion is not robust, not leading to the desired results or is even not possible in many cases. The aim of this research is to mimic the way of thinking and logic of the engineer and to make decisions based on component and sub-assembly recognition. By identifying components and assigning them to categories, the developed recognition framework allows streamlining and specifically optimizing the process for each category, making it a more robust and comprehensible approach.
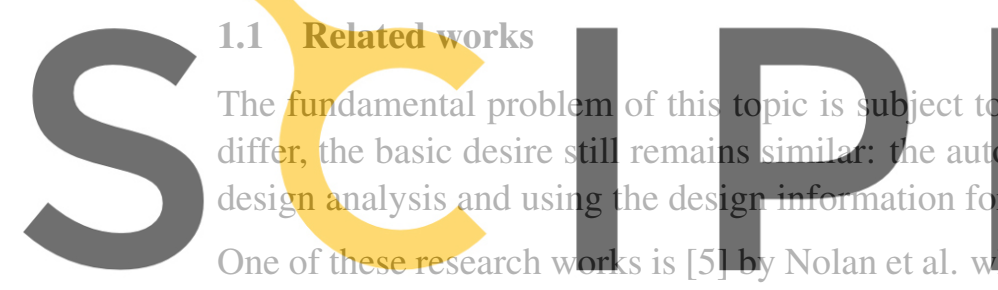

One of these research work
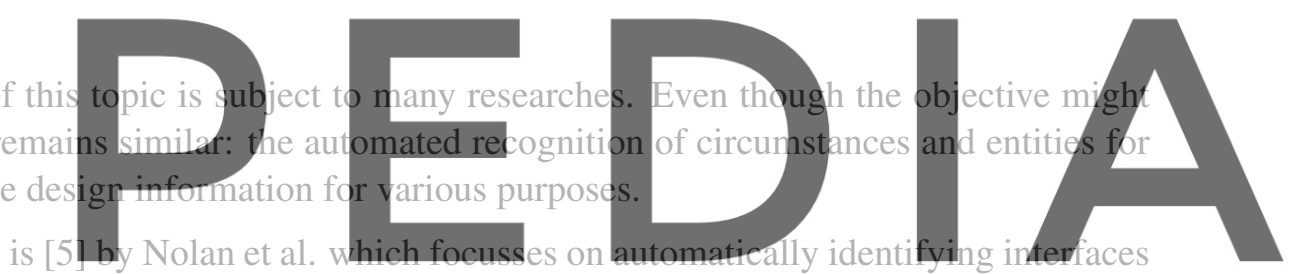

required for subsequent simulation or important geometric entities. The application of this idea is shown

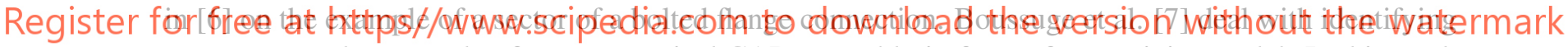
structures on the example of an aeronautical CAD assembly in form of a root joint model. In this work the identification of interfaces is also substantial in order to apply correct boundary conditions, e.g. bolt connections, and geometric pre-processing. This idea is also addressed by Shahwan et al. in [8] who make use of behavioural reasoning from components interfaces to components functions for FE analyses.

From a general point of view, the engineer is dealing with this task by visually identifying interfaces and components, deriving functions of parts using engineering and general knowledge, evaluating potential representations in the simulation on the basis of experience and applying the boundary conditions manually. The key idea behind this is identifying three dimensional objects which is also a common topic in other fields like computer graphics. Several approaches to recognize 3D objects have been developed in the last years. An exemplary technique is the analysis of 3D parts using ontology where the face adjacency graph is the major object descriptor [9]. Similar to this, Cicec et al. [10] also work on part recognition by accessing the relations between geometric entities like faces. Di Stefano et al. [11] make also use of topology and feature semantics in order to analyse a given CAD part. The features to be recognized in this research are mainly from the machining domain such as turning and milling features. Tangelder et al. [12] and Iyer et al. [13] present an overview of common approaches for 3D shape 
analysis.

Introducing part context means elevating the perspective level from single components to an assembly. This has been investigated by Deshmukh et al. in [14] where a content-based search technique is developed in order to improve reusability in the design process. Mating and joints are describing the relationships between parts which shall serve as criteria for design recognition. Chen et al. [15] follow a similar idea of analysing components by also considering a kinematic context by interface evaluation. The product structure and the relations between different parts are analysed afterwards based on this. In [16] Lupinetti et al. target the objective of reusing existing design knowledge. For this reason, the attempt is to extract information and knowledge from geometry and associated metadata and to use this data for matching similar models from a database. Lupinetti et al. further investigates in [17] the creation and use of this enriched assembly model. A survey and future challenges of this approach are given in [18]. The method described by Hasan et al. in [19] works with the SolidWorks API to access geometric information to gather assembly relation information. Similar to previous approaches, a semantic assembly model is built using the gathered information. This assembly model uses separated data in form of geometrical semantic data represented by form features such as holes and slots and assembly constraints like alignments and joints.

In summary, most of these works share the same idea but none of them is meeting the challenge of this research. Geometrical designs are changing, thus making a topology-related shape matching less reasonable. Furthermore, besides interface analysis more specific information is required to provide an adequate guideline to the automated CAD-to-CAE process. For this reason, this research has been done

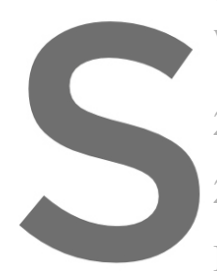
which deals with linking

2 Recognition framework

2.1 Target components

In the example of an aengine structural assemb
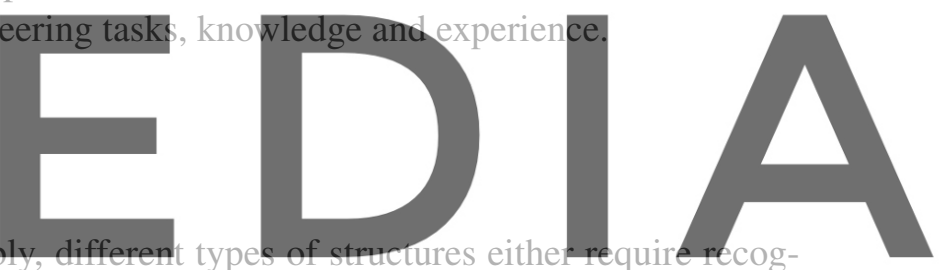

nition or their identification can benefit the analysis. The application of boundary conditions in complex

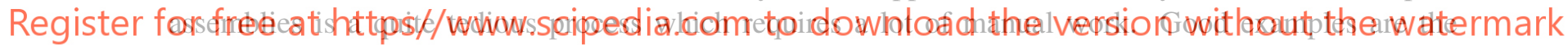
multiple bolt connections present in these assemblies. Due to complexity coming along with contact simulations, these are commonly replaced by surrogate constructs which have to be applied manually to the related entities. Apart from increasing the process efficiency, an automation entails also an advantage for the post-processing due to having direct access to the connections, thus FE nodes and elements.

Clusters of these bolts can be found at component flanges. Especially those bearing the main load path are decisive for the behaviour of the whole engine, thus depict an important interface, also for postprocessing and deriving flange loads.

Another important interface is the connection between rotary and static sub-assemblies. Due to rotordynamic effects, both systems are usually treated and analysed separately. Nevertheless, the transfer of forces and loads between both systems has to be maintained. For this reason, the recognition of the bearing location as well as identifying rotary and static structures is of advantage for the automated understanding of the whole engine system.

As a result of weight and dimensional restrictions, many components in aero engine assemblies possess a thin-walled topology. This circumstance is the reason for simplifications using dimensional reduction 
entailing an attractive potential for complexity reduction while maintaining model accuracy. Similar to this, long and slender structures such as struts are also representable by lower dimensional FE surrogate models what in turn emphasizes the benefit of their identification.

\subsection{Overview of the approach}

The fundamental of this approach is the aim to mimic the process an engineer's way of thoughts during the development of a structural whole engine model from a geometry. First it is to understand how a human is accessing the information and in which way this information is processed as such to derive conclusions from the present geometry. Since aero engine structures depict highly optimized assemblies, standardization is only present in smaller parts such as bolts. Other components are adapted and optimized to the current environment, consequently altering their shape and topology. For this reason, a purely shape and topology based approach is rather not robust or not achieving the target.

The advantage of the approach presented is that, besides topology, a function, context and positioning evaluation is supporting the identification. The function that a component is fulfilling in an assembly will remain the same no matter how the shape is changing. The same idea applies to the context analysis.

Depending on the component category, the weighing of the different identification criteria will differ. Small standardized parts such as bolts (section 2.5) can be identified purely by shape where as other components such as bearings (section 2.4.1) also put emphasis on the function aspect. Starting from basic geometric entities, higher-level components can be recognized based on the context given.

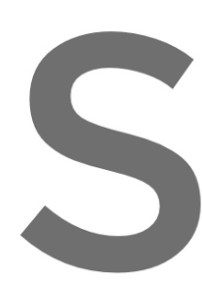

After having identified

tification and categorizatic accessed directly for later

\section{Register for}

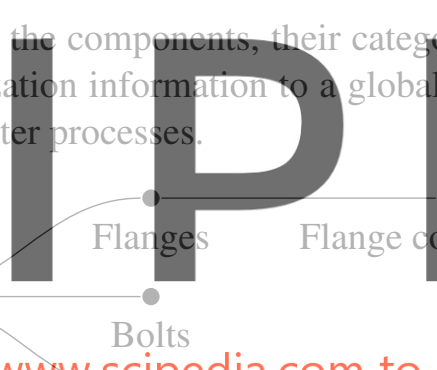

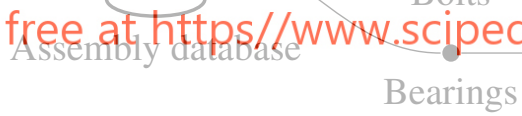
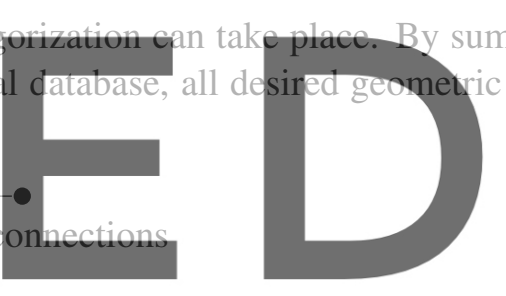

Casing

Casings

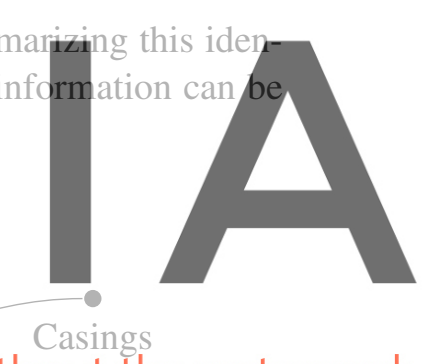

free at https//www.sciped
Bearings

Rotary groups

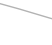

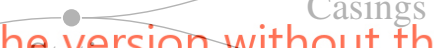

Beam structures

Rotary

Figure 1: Database scheme

\subsection{Interferences and interfaces}

The interfaces of different components or substructures are of major importance to describe their function. To set-up a network of interfaces of all assembly components, the first step is to develop methods to access this interface information from the geometry assembly. After having identified interfaces, it is important to evaluate the type of interface to derive kinematic conditions. Especially planar and cylindrical interfaces are of major importance and therefore described in this paper.

Evaluating potential contact faces is a performance-greedy process, So to improve the performance, a bounding box filter algorithm has been used first. The interference methods provided by NX Open only retrieve interfaces which are exactly touching. However, due to various reasons, in engineering 
CAD assemblies small tolerances might occur. To consider these, another algorithm has been introduced which uses a shortest distance method and uses $\varepsilon$ as a tolerance parameter. The next issue is to identify a touching condition between two entities for which further sub-routines have been developed. A pseudocode sketching this process is shown in algorithm 1 . The sum of all identified interfaces and interferences are organized in an interface and interference network which is basis for later analyses.

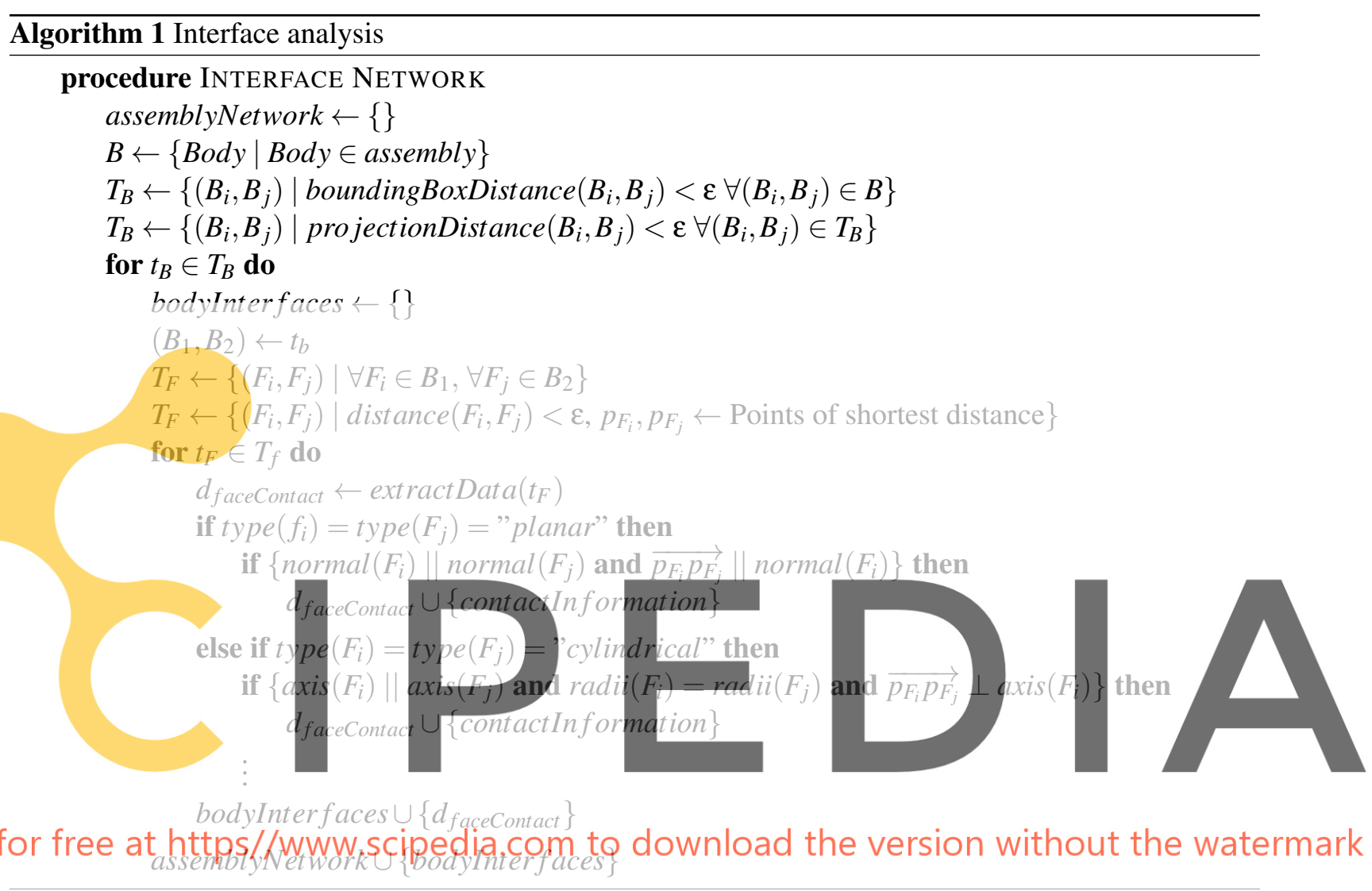

\subsection{Entities}

\subsubsection{Bearings}

One of the entities of major importance for model understanding is the category bearings. In order to identify bearings, the function plays a major role. Bearing rings and the connection to the components can differ in shape and form drastically, but the function of a bearing will remain the same: it represents the connection of two rotating parts where the rotating speed of one structure can also be zero, so depict a static structure. Generally, it consists of an outer and an inner ring, multiple bearing bodies and an optional bearing cage. Consequently, its interference representation can be illustrated in a simplified form as in figure 2 .

To extract these systems from the assembly, the bearing bodies are first put into focus. In the course of this research, the focus is set on the most common bearing body types: ball and roller bearings. Since the shape of them in turn will not change due to standardization, the recognition via shape is a 


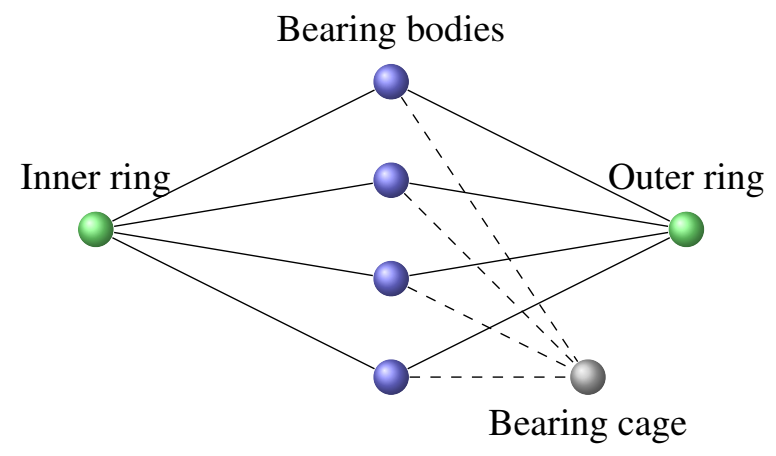

Figure 2: Bearing scheme

reasonable approach. Consequently, an algorithm is filtering potential bearing bodies using topology and grouping them based on shape properties. The information stored in the interface network further groups them based on their interfaces. Thereof, the derived neighbour bodies are evaluated regarding quasi-axisymmetry. For a proper assignment of inner and outer ring and optional bearing cage from the neighbouring parts, a ray tracing algorithm is applied in addition to the interface information. Based on the gathered information, the bearing entities can be set up. Further information as the bearing axis can be calculated from the bearing bodies, the boundary conditions of the bearing can be derived from the interfaces between bearing body and ring(s) and the bearing shape properties are set by the first body

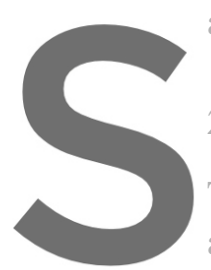
analysis.

\subsubsection{Flanges}

The next entity of importance for an important influence on
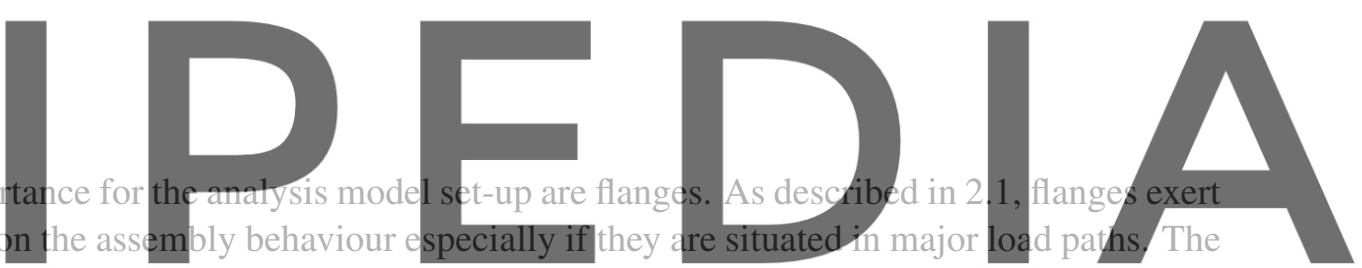

Register for free at he mostcommon flang is the flange gonnecting two components alont a circumference with bolts (figure $3 a)$. Due to assembly reasons, it is also common that a component is divided into two parts. This means also splitting the flange (figure 3b) and adding an additional flange to assemble both longitudinal splits (figure 3c).

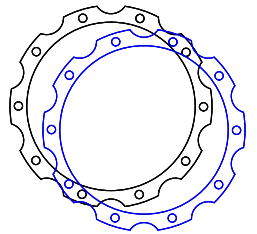

(a) Standard flange

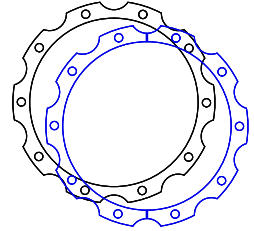

(b) Splitted flange

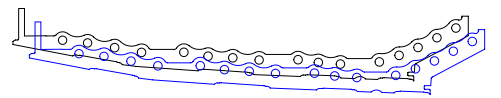

(c) General flange

Figure 3: Flange types

The major aspects for identifying flanges are the function, which is the connection between two or more components, and shape criteria like flange height, thickness and optional matching flange holes for bolt connections. Consequently, the starting point is the planar interface information from the interface 
network. These planar interfaces have to be evaluated further to match the flange shape requirements. To distinguish between the three types of flanges mentioned above the interference network information is used. If two faces share a planar interface with a single face while both combined match the topology of the single face, the conditions for a split flange are given. Otherwise, if two single faces are coincident and they share a topological centric hole, as for example for the gas path, an engine "standard" flange is present. The criteria for the third type is given if two faces share the same topology without being quasi-axisymmetric nor sharing a centric hole from a topological point of view.

The next step is to project all related faces to a shared plane created by the face normal. By this, the touching condition is analysed in more detail and furthermore, information concerning maximum and minimum flange height is extracted. The flange thickness is defined by the distance to the rear side of the flange. In order to derive the rear faces, a similar approach to algorithm 1 is applied using face properties and a distancing approach. From this set of faces, the closest within a tolerance define the corresponding rear face or faces. The last criterion is the distribution of shared flange holes. The edges defining the holes are derived via a topological analysis of the faces. Starting from these edges, crawling through adjacency to the rear flange face yields the whole chain of entities required for bolt connections, e.g. hole inner faces. Having extracted these flange holes, they can be matched to the partner flange to create a programming structure describing a flange hole connection.

All this information and further information regarding flange chains is stored in the assembly database within structured classes to allow an easy access for further processing.

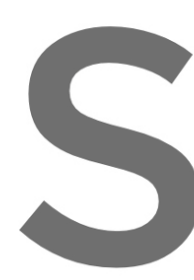

2.5 Bolts

Staying in the topic of entity in the assembly. assemblies designed for hole diameter and further
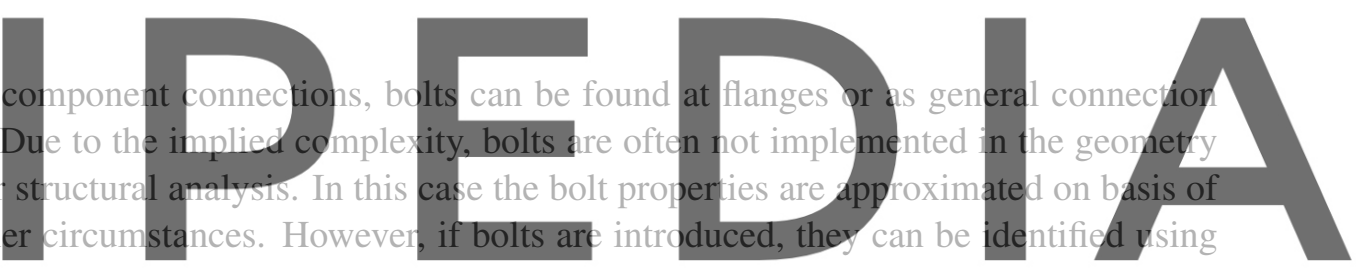

only shape criteria due to their standardization. In terms of standardization, the number of different types

Register for holts is often kept to a minimum. As exemplary application case in this research the analysis of a double-hex bolt is put into focus.

The major features that are crucial for bolt recognition is on the one hand the thread and on the other hand the bolt or nut head form. In complex assemblies the thread is also modelled in a symbolic way to keep the complexity as low as possible. Consequently, the approach used in this recognition framework is focussing on the head form. An exemplary bolt and its sorted face adjacency graph are shown in figure $4 \mathrm{a}$ and $4 \mathrm{~b}$. The face formation shaping the head is defined by standardized relations and properties which can be retrieved from face data and the face adjacency graph, shown in green. Since both bolt and nut contain this relation structure, they are distinguished using a ray tracing technique along the center line. After having identified the target head faces for the tool, the remaining body can be analysed using topological operations in order to extract further bolt properties.

In the presented recognition framework, if bolts are recognized, their properties are used for the FE surrogate model set-up otherwise these properties are approximated on basis of the present geometry conditions. 


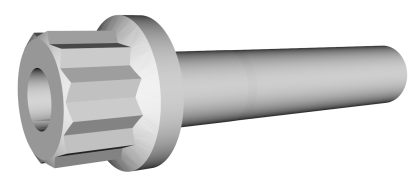

(a) Double-Hex bolt

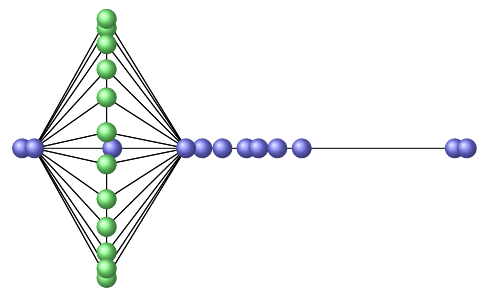

(b) Topology network

Figure 4: Bolt topology

\subsection{Higher-Level entities}

\section{Rotary groups}

As shown in figure 1 in section 2.2, the bearings can be used due to their function to build up a context for retrieving sub-assembly groups. In combination with the interference network, an algorithm has been developed to assess connected paths while having the bearings as interfaces to other paths. Working on these interfaces, it is possible to combine the gained information to separated groups and the related inner and outer bearing interfaces as shown in scheme 5 .
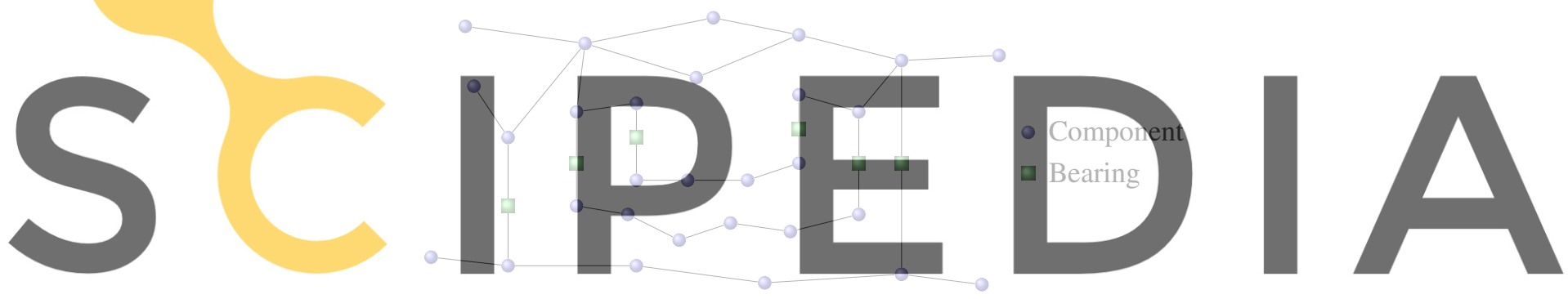

Register for free at https//www.scipedigure 5: Rotary group graph to download the version without the watermark

To further exploit this information, the next step is to transfer the knowledge of aero engines to these groups. In aero engines, the main rotary assemblies are wrapped by the static structural system, meaning that the stationary system only has inward interfaces to the rotating sub-assembly. Furthermore, these rotary structures and especially the low-pressure rotor depict comparably slender structures along the center axis in all standard aero engine architectures. With a sub-assembly analysis and the information derived from the rotary group identification this logic can be transferred to the digital recognition. The results lead to a clear specification of low-pressure, optional intermediate pressure and high-pressure rotor assemblies as well as to the stationary structure. This information in turn could serve as further identification aspects for rotor entities as for example discs, the burner section and consequently also for compressor and turbine groupings.

\section{Beam structures}

From these stationary structures, another category of interest for identification are beam-like structures. Due to their nature, a dimensional reduction to $1 \mathrm{D}$ FE elements can bring performance improvements 
while keeping the implied error relatively low. Recognizing these components is relatively straightforward for the human eye and brain but recognition on the basis of computational data is a more delicate problem. The technique applied for this category is utilizing general mass properties as for example inertia axes. On the basis of these axes, an oriented bounding box is set up to measure the maximum dimensions of the present part. The presented recognition framework then sets the dimensions into relation to evaluate the component and to specify it as beam-like structure. The next step in a subsequent process is the retrieval of reasonable cross-sections for the beam FE elements.

\section{Casings}

A similar drive is leading to the specification of the casing category. As mentioned in section 2.1, they are of special interest due to their mainly thin-walled topology. Casings by definition imply the fact that they are wrapped around something, in this case they surround the gas path. This fact is leading to a quasi-axisymmetric set-up and the direction being collinear to the engine axis. Topological data and geometric entities as for example faces are providing information to identify these characteristics. A high percentage of faces relative to their area being directed along engine axis is an access point for the recognition framework. Furthermore, a mathematical construct similar to the bounding box but on basis of cylindrical coordinates is developed and applied to describe the cylindrical properties of the casing structure. In a subsequent process, the identified potential casings structures are further analysed towards an automated transfer to the CAE environment.

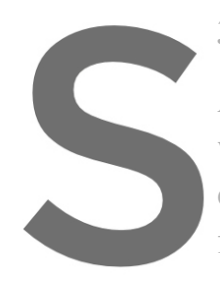

3 Application

After all these methods ware Siemens NX, the of this work, an exempl figure 6a. The interface $\mathrm{n}$ ave been implemented us
rst application uns have
network described in secti

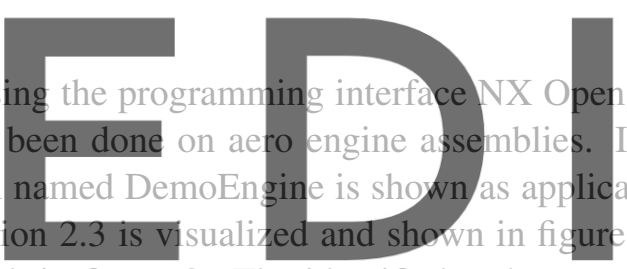
In the
ication
ure $6 c$. T a closer look reveals structures similar to the graph in figure 2. The identified and categorized rotary Register for free at hte by fhe framework are depicted in fiqure 6h. A graphical user interface (GUI) is guiding the user to the categories and simple action buttons identify and reveal the target components and categories. On account of the developed techniques, the recognition framework is able to set-up a database for various purposes as for example CAD pre-processing and boundary condition application. A performance overview is given in table 1 . The approach which also takes modelling tolerances into account is more computational expensive due to several reasons (method 2). However, this method introduces an improved robustness to the process. As a result of the internal kernel of Siemens NX, thus NX Open, all methods are limited to a single CPU core. Consequently, a multi-CPU realization could improve the performance drastically. Nevertheless, it has to be kept in mind that for example manually identifying all bearings and all potential flange connection along with connection holes remains a more time-consuming task for the engineer.

Table 1: Performance evaluation on DemoEngine model

\begin{tabular}{ccccc}
\hline Model & \# Parts & \# Interferences & Method 1 [s] & Method 2 [s] \\
\hline DemoEngine & 279 & 475 & 128.8 & 300.8 \\
\hline
\end{tabular}




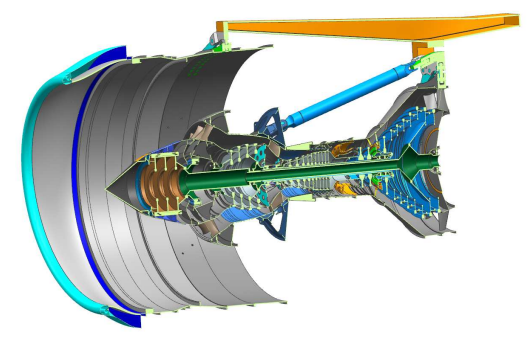

(a) Aero engine model (DemoEngine)

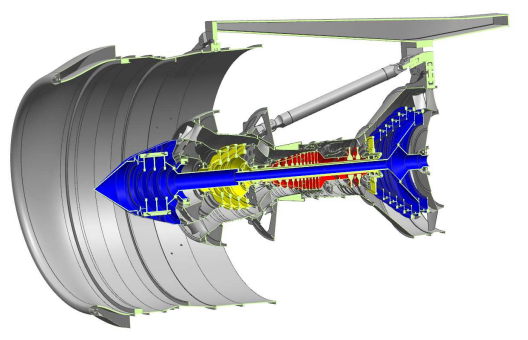

(b) Rotary groups identification
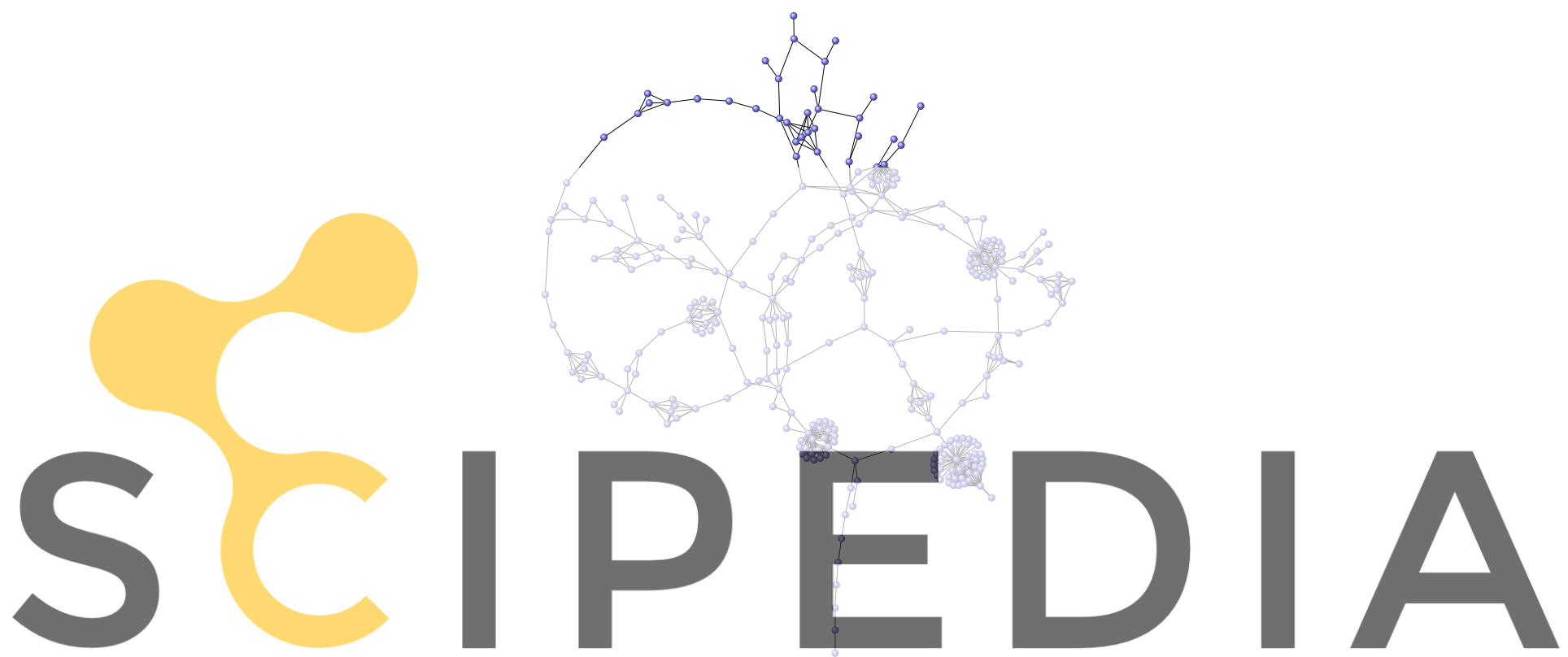

Register for free at https//www.scipedia.commtedpamblpad the version without the watermark

Figure 6: Application of the recognition framework

\section{Conclusion}

This work presents a method to mimic the engineer's work in the process of creating a simulation model for present geometry assemblies. The fundamental idea is that the steps taken are defined and guided by a precedent recognition and evaluation. Especially realizing and transferring the logics and way of thoughts behind these steps to computer language has been identified as the key problem. In the scope of this work, the application case aero engine with specific entities of interest for structural analyses is put into focus. However, the main concept can be applied to various fields and also to more wider field of target components. In summary, the results show that automating the process of geometry identification can substantially improve process efficiency and is also able to maintain and provide reasonable data to later analyses. With the developed recognition framework, a fundamental starting point for applying category-specific optimized methods and a streamlined process is given. 


\section{References}

[1] Okba Hamri and Jean-Claude Léon. "Interoperability between CAD and Simulation Models for Cooperative Design". In: Methods and Tools for Co-operative and Integrated Design (2004), pp. 451-462. DOI: 10.1007/978-94-017-2256-8_38.

[2] Okba Hamri et al. "Method, Models and Tools for CAD-CAE Integration". In: Recent Patents on Mechanical Engineering 3.2 (2010), pp. 106-130. ISSN: 1874477X. DOI: 10 . 2174 / $1874477-$ x11003020106.

[3] Sang Hun Lee. "A CAD-CAE integration approach using feature-based multi-resolution and multiabstraction modelling techniques". In: CAD Computer Aided Design 37.9 (Aug. 2005), pp. 941955. ISSN: 00104485. DOI: $10.1016 /$ j.cad.2004.09.021.

[4] Atul Thakur, Ashis Gopal Banerjee, and Satyandra K. Gupta. A survey of CAD model simplification techniques for physics-based simulation applications. 2009. DOI: $10.1016 / \mathrm{j}$. cad. 2008 . 11.009.

[5] Declan C. Nolan et al. "Defining simulation intent". In: CAD Computer Aided Design (2015). ISSN: 00104485. DOI: $10.1016 / \mathrm{j}$.cad.2014.08.030.

[6] Declan Nolan et al. "Automating analysis modelling through the use of simulation intent". In: NAFEMS World Congress ; Conference date: 09-06-2013 Through 12-06-2013. June 2013.

[7] Flavien Boussuge. "Idealization of CAD Assemblies for FE Structural Analyses". dissertation. July 2014.

[8] Ahmad Shahwan et al. "Qualitative behavioral reasoning from components' interfaces to components' functions for DMU adaption to FE analyses". In: CAD Computer Aided Design 45.2 (2013), pp. 383-394. ISSN: 00104485. DOI: 10.1016/j.cad.2012.10.021.

[9] Mingcong Huang et al. "Three-dimensional CAD Model Retrieval Algorithm Based on Ontology". In: Procedia CIRP 56 (2016), pp. 590-593. ISSN: 22128271. DOI: 10.1016 / j . procir. 2016.10.116.

[10] Adem Çiçek and Mahmut Gülesin. "A part recognition based computer aided assembly system". In: Computers in Industry 58.8-9 (Dec. 2007), pp. 733-746. ISSN: 01663615. DOI: 10.1016 / j . compind.2007.02.007.

[11] Paolo Di Stefano, Francesco Bianconi, and Luca Di Angelo. "An approach for feature semantics recognition in geometric models”. In: CAD Computer Aided Design 36.10 (Sept. 2004), pp. $993-$ 1009. ISSN: 00104485. DOI: $10.1016 / \mathrm{j}$.cad.2003.10.004.

[12] Johan W.H. Tangelder and Remco C. Veltkamp. "A survey of content based 3D shape retrieval methods". In: Multimedia Tools and Applications 39.3 (Sept. 2008), pp. 441-471. ISSN: 13807501. DOI: $10.1007 / \mathrm{s} 11042-007-0181-0$.

[13] Natraj Iyer et al. "Three-dimensional shape searching: State-of-the-art review and future trends". In: CAD Computer Aided Design. Vol. 37. 5 SPEC.ISS. Elsevier Ltd, Apr. 2005, pp. 509-530. DOI: $10.1016 / j . c a d .2004 .07 .002$.

[14] Abhijit S. Deshmukh et al. "Content-based assembly search: A step towards assembly reuse". In: CAD Computer Aided Design 40.2 (Feb. 2008), pp. 244-261. ISSN: 00104485. DOI: 10.1016/ j. cad.2007.10.012.

[15] Xiang Chen et al. "A flexible assembly retrieval approach for model reuse". In: CAD Computer Aided Design 44.6 (June 2012), pp. 554-574. ISSN: 00104485. DOI: 10.1016/ j . cad.2012. 02. 001. 
[16] Katia Lupinetti et al. "Multi-criteria retrieval of CAD assembly models". In: Journal of Computational Design and Engineering 5.1 (2018), pp. 41-53. ISSN: 22885048. DOI: $10.1016 / j$. jcde . 2017.11.003.

[17] Katia Lupinetti et al. "Content-based multi-criteria similarity assessment of CAD assembly models". In: Computers in Industry 112 (Nov. 2019). ISSN: 01663615. DOI: 10.1016 / j . compind. 2019.07 .001$.

[18] Katia Lupinetti et al. "Content-based CAD assembly model retrieval: Survey and future challenges”. In: Computer-Aided Design 113 (2019), pp. 62-81. DOI: 10.1016/ j.cad.

[19] Baha A. Hasan, Jan Wikander, and Mauro Onori. "Assembly design semantic recognition using solid works-API". In: International Journal of Mechanical Engineering and Robotics Research 5.4 (2016), pp. 280-287. ISSN: 22780149. DOI: 10.18178/i jmerr. 5.4 .280-287. 